\title{
KMOS: an infrared multi-integral field spectrograph for the VLT
}

\author{
R.M.Sharples ${ }^{\mathrm{a}}$, R. Bender ${ }^{\mathrm{b}, \mathrm{c}}$, R. Hofmann ${ }^{\mathrm{c}}$, R. Genzel ${ }^{\mathrm{c}}$ and R.J. Ivison ${ }^{\mathrm{d}}$ \\ ${ }^{a}$ Department of Physics, University of Durham, Durham DH1 3LE, UK \\ ${ }^{b}$ Universitätssternwarte München, Scheinerstraße 1, D-81679, München, Germany \\ ${ }^{\mathrm{c}}$ MPE, Giessenbachstraße 1, 85748 Garching, Germany \\ ${ }^{\mathrm{d}}$ Astronomical Technology Centre, Royal Observatory, Blackford Hill, Edinburgh EH9 3HJ, UK
}

\begin{abstract}
We describe a proposed $2^{\text {nd }}$ generation instrument (KMOS) for the ESO VLT which will deliver a unique multiple deployable integral field capability in the near-infrared (1-2.5ì m). The science drivers for such an instrument are presented and linked to the functional specification. The overall instrument concept is discussed in the context of two proposed solutions for delivering a deployable integral field capability. Detailed discussions of these two approaches, and ongoing prototype studies, are described in separate papers.
\end{abstract}

Keywords: Integral field spectrographs, multiple object spectroscopy, infrared spectrographs

\section{INTRODUCTION}

In the last decade, cosmology has entered a golden age. Large optical imaging and redshift surveys (e.g. APM, 2dF, SDSS), supernova search projects, cosmic microwave background experiments (e.g. COBE, BOOMERANG) and X-ray surveys (e.g. ROSAT, XMM) have been providing increasingly stringent constraints on the basic parameters of the world model. Within the next few years, several experiments exploring the microwave background will deliver precise values for the fundamental cosmological parameters $\mathrm{H}_{0}, \Omega_{0}, \Lambda$ and the primordial power spectrum of fluctuations. Furthermore, currently ongoing or planned optical/IR spectroscopic surveys and gravitational lensing studies with the CFHT, VST, VIMOS, DEIMOS and other instruments will constrain the evolution of the luminosity function of galaxies, their star formation rates and their large scale clustering as well as the evolving power spectrum of the dark matter distribution with increasing accuracy.

With the geometry of the universe finally settled and the global parameters of galaxies approximately known, testing the hierarchical paradigm, i.e., understanding the actual physical processes governing the formation of galaxies, the growth of their masses and the origin of their structure and morphology, will become the major challenge of astrophysical research in the next decade. Coupled with the fundamental issue of early galaxy evolution is the question of how massive black holes formed and how their evolution is connected to that of their host galaxies. Although the last few years have also seen significant progress on these questions, the most important issues are still open. Two requirements follow if we want to make progress in answering these questions:

First, it is essential to obtain spatially resolved, spectroscopic information about galaxies at medium and high redshift - if possible using adaptive optics to improve the spatial resolution. In order to probe statistically meaningful samples of objects, it is necessary that data on a large number of objects are obtained simultaneously. Sampling galaxy kinematics also requires moderately high spectral resolving powers $(\lambda / \Delta \lambda>3000)$. The currently tightest constraints on the evolution of galaxies at $z<1$ could not have been obtained without high resolution imaging (mostly with HST) and spatially resolved spectroscopy under very good seeing conditions. The latter has yielded rotation curves, velocity dispersion and stellar population profiles which have allowed the accurate analysis of the evolution of the Tully-Fisher, Fundamental Plane and Mg- $\sigma$ relations, as well as the nature of E+A galaxies. These measurements confirm that spirals and ellipticals with present day masses and morphologies were largely in place already at $\mathrm{z} \sim 1$. Only irregulars show significant evolution at low redshifts. Galaxies at higher redshifts typically have complex morphologies and their kinematic major axes cannot be determined a priori from images. As indicated by the recent serendipitous discoveries of Lyman-á 
emitters, two-dimensional, imaging spectroscopy is also especially valuable for finding and exploring objects at the highest redshifts.

Second, it is important to extend this multi-object survey capability to the near infrared. Up to now, ground based extragalactic research has been driven mainly by optical observations. However, optical observations alone are not able to study the assembly of the present-day galaxy population much beyond $\mathrm{z} \sim 1$ because crucial spectral features for the analysis of their old stellar populations (e.g. via the 4000Å break) and their kinematics (key emission lines like [OII] and $\mathrm{H} \alpha$ ) are shifted into the near-IR. At $\mathrm{z} \sim 1-2$, infrared spectroscopy will permit analysis of the masses, the stellar populations, and star-formation rates of galaxies. At $\mathrm{z}>2$, infrared observations provide estimates of masses and metallicities of galaxies from their rest-frame optical emission lines. At z>6, prominent UV emission lines, such as HeII and Lyman- $\alpha$ move into the near-IR. Finally, many crucial events in the life of a galaxy, like mergers, or the outburst of nuclear activity are enshrouded in dust and can only be observed in the infrared. For observations of key spectroscopic indicators throughout the $\mathrm{z} \sim 1-3$ redshift range, infrared spectroscopy should cover the $\mathrm{J}, \mathrm{H}$ and $\mathrm{K}$ windows, thus requiring a cryogenic spectrograph.

\subsection{NUMBER DENSITIES OF GALAXIES IN THE NEAR INFRARED}

Because the observation of high redshift galaxies is very time consuming, an instrument to exploit these scientific challenges should have a multiplex capability adequate for the surface density of observable galaxies. The unvignetted Nasmyth field at the VLT has a diameter of approximately 7.2 arcmin. For a $50 \operatorname{arcmin}^{2}$ field of view, the total number of sources with $\mathrm{K}=20$ and accessible to spectroscopy (see below) amounts to several hundred sources. However, the vast majority of these are relatively uninteresting low-redshift objects, and they can be identified with optical and infrared imaging surveys (via the photometric redshift technique) or optical spectroscopy. The number density of objects with interesting luminosities and redshifts, e.g. Extremely Red Objects (EROs), x-ray sources and dusty starbursts, typically amounts to about 10 to 100 galaxies per $50 \operatorname{arcmin}^{2}$ field, depending on clustering (see Fig. 1).

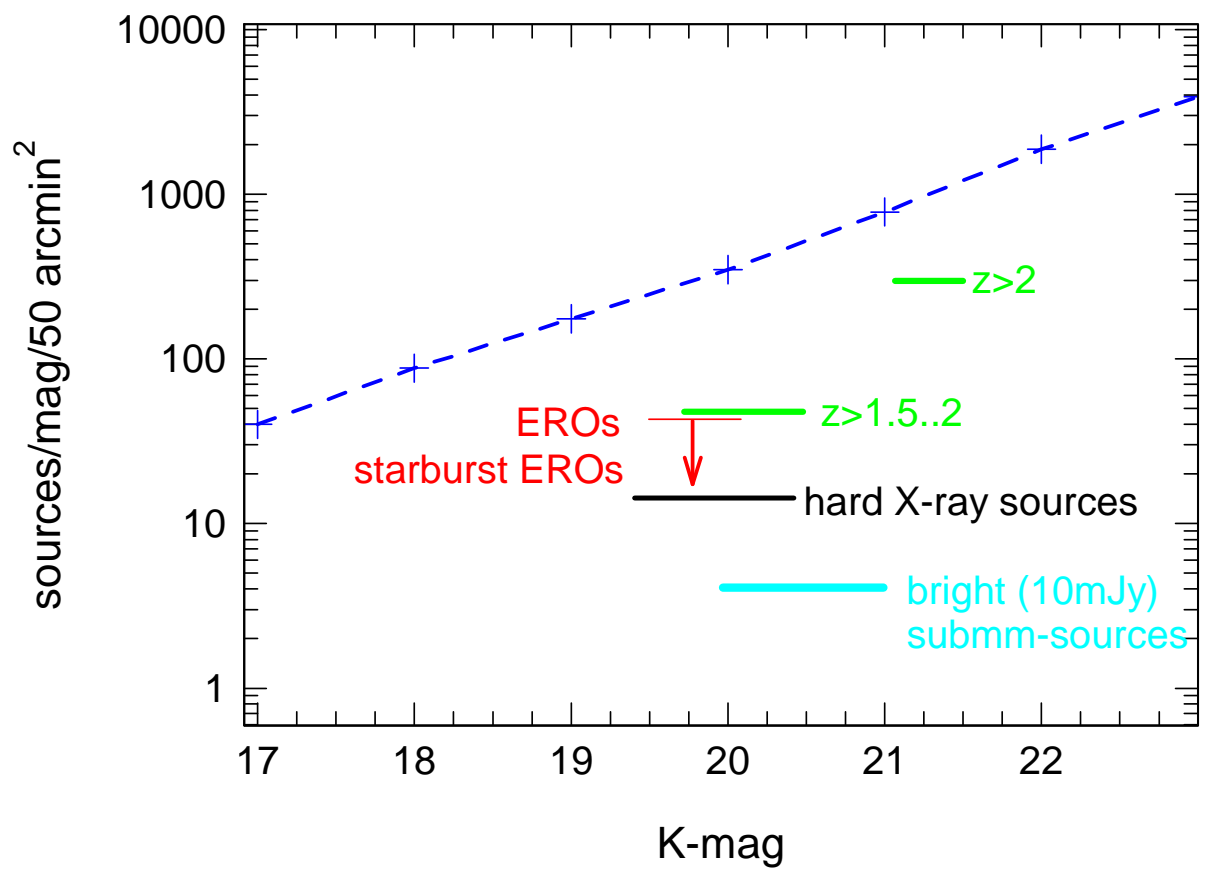

Figure 1. Source surface densities as a function of K-band magnitude. The blue dashed curve and crosses denote the total K-band source counts ${ }^{1}$. The fractions of those sources at high redshift ${ }^{2,3,4}$ are denoted by green bars. The source density of $2-10 \mathrm{keV}^{2}$ sources ${ }^{5}$ $\left(>210^{-15} \mathrm{erg} / \mathrm{s} / \mathrm{cm}^{2}\right)$ is indicated as a black bar. The surface density of extremely red objects ${ }^{6}$ (EROs: R-K $>5$ ) is marked by a red bar, with an arrow denoting the fraction of EROs that are likely dusty starbursts (rather than early-type galaxies) ${ }^{7}$. The source density of bright $\left(\mathrm{S}_{850 \mathrm{~mm}}=10 \mathrm{mJy}\right)$ submm sources ${ }^{8}$ is indicated by a light blue bar. 


\subsection{THE CASE FOR DEPLOYABLE INTEGRAL FIELD UNITS}

Since galaxy morphologies become more complex with increasing redshift (Figure 2), it is important to obtain more than just one-dimensional information about these objects, i.e., one needs spatially resolved spectroscopy using integral field units (IFUs). In addition to their mapping capability, IFUs have other advantages compared to slitlets: centering requirements on the objects are relaxed, there are no slit losses, spatial pixels can be binned post-factum to obtain optimal signal-to-noise ratio, no assumption about a kinematic symmetry axis (if existent) has to be made before the observation, and there is significant serendipitous discovery potential for faint emission-line companions. State of the art, fibre or slicer based IFUs have throughputs (60-80\%) that are close to those of slit masks so that IFUs are superior to slit spectrometers even for compact sources.
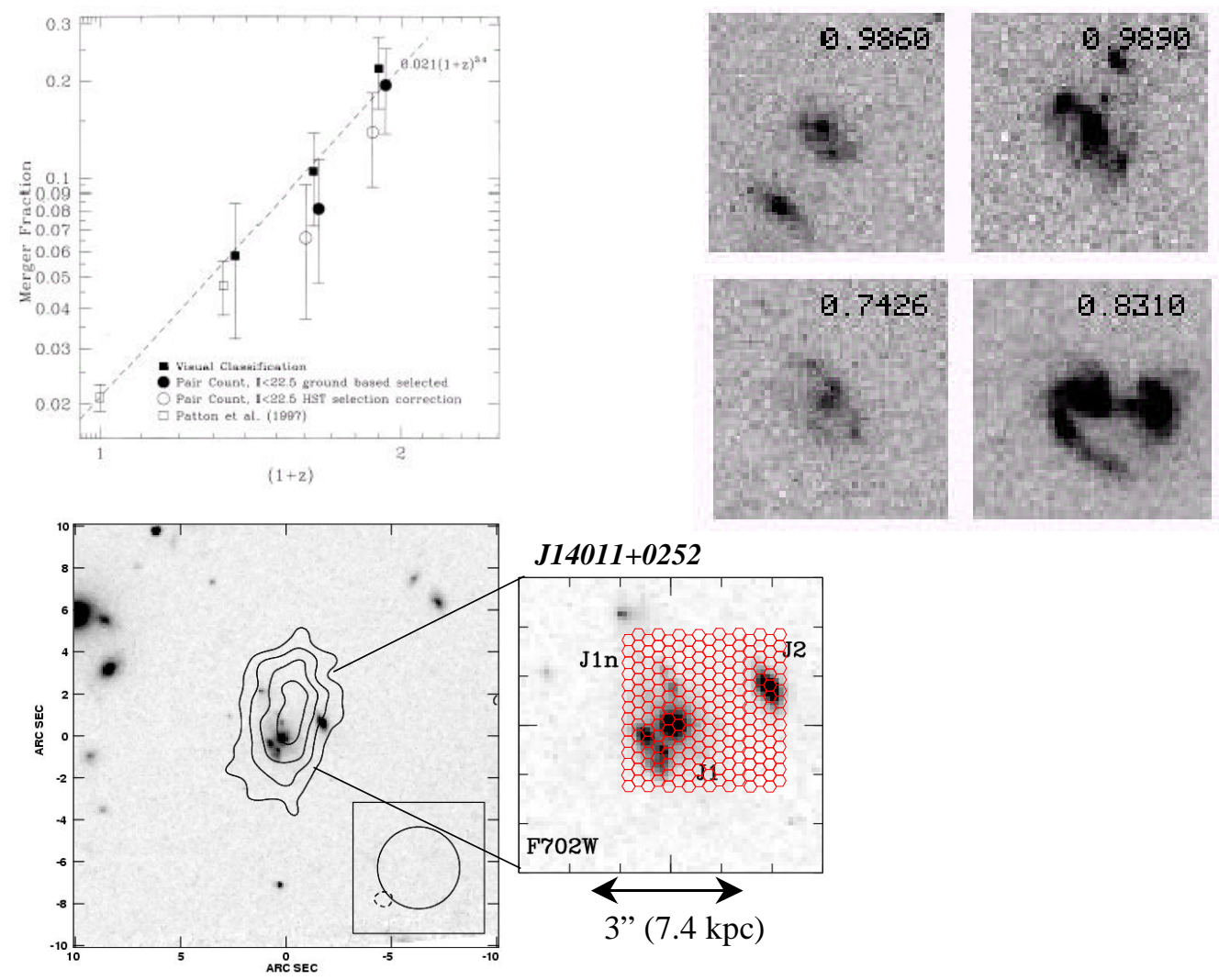

Figure 2. These figures demonstrate the spatial complexity of high-z galaxies and the need for two-dimensional imaging spectroscopy Top Left: The evolution of the visually estimated mergers (filled squares) and spectroscopically confirmed pairs (circles) ${ }^{9}$. Many high-z galaxies are mergers, or lie in complex fields. Top Right: Examples of HST images of galaxies visually classified as mergers. The redshifts are indicated in the top corner and each image is 5" x 5". Bottom: Morphology of the z=2.56 SCUBA submillimeter galaxy SMM14011+0252 ${ }^{10}$. The popout image shows the F702W HST image of the galaxy, with a 0.2 " per pixel IFU superposed. The left image shows the HST image with CO millimetre-line emission contours superposed. SMM14011+0252 clearly is a complex object, with a number of UV bright spots spread over $>10 \mathrm{kpc}$, and embedded in an extended cold dust/gas distribution.

One subtle advantage of an IFU compared to a slit is in the amount of light from an object that can be used in constructing an integrated spectrum. An IFU can more fully sample the light profile of an object than a slit. This is demonstrated by the simulation results in Figures 3 and 4. 


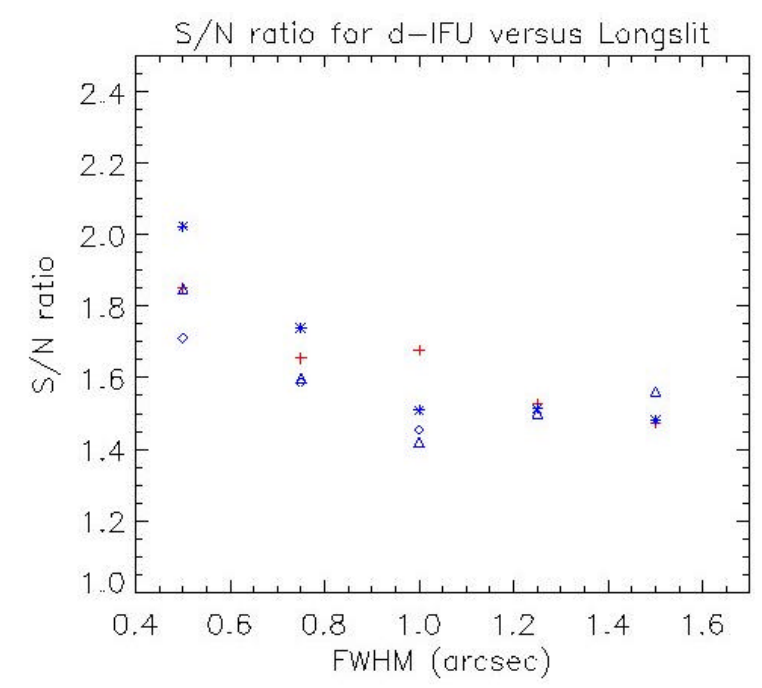

Figure 3. Simulation of the signal-to-noise advantage provided by an IFU compared to a slit spectrograph. In these simulations, both spectrographs have the same characteristics of $R=\lambda / \Delta \lambda=5000$ for 2 pixel sampling. The pixels size is 0.2 arcseconds and the IFU field of view is 2.8 " $\mathrm{x} 2.8$ ", whilst the slit is 1 " wide and 10 " long. The resolution of the IFU fed spectrograph is 5000 , whilst for the slit it is only 2000 due to the projected width (5-pixels) of the slit. Of course it is possible to conserve resolution of $\mathrm{R}=5000$ with a 0.4 " slit width, but the light losses would then be even more severe. The light profiles of these simulations assume an elliptical $R^{1 / 4}$ profile for a range of half-light radii and Gaussian seeing profiles. Real galaxies of course do not follow such a clean profile in any sense other than azimuthally. So whilst this model is idealized, it provides a reasonable estimate of the advantage an IFU might provide by using the light more efficiently than a single slit. The following $\mathrm{R}^{1 / 4}$ radii were chosen: 0.25 "-cross, 0.5 "-asterix, 0.75 "-triangle, and 1.0 "diamond. These profiles were then convolved with Gaussian seeing profiles of various FWHMs. In the best seeing conditions and most compact objects, the IFU provides a significant advantage over a slit. For the sizes of typical high redshift galaxies and Paranal seeing conditions, the advantage in $\mathrm{S} / \mathrm{N}$ is about a factor of 2 .
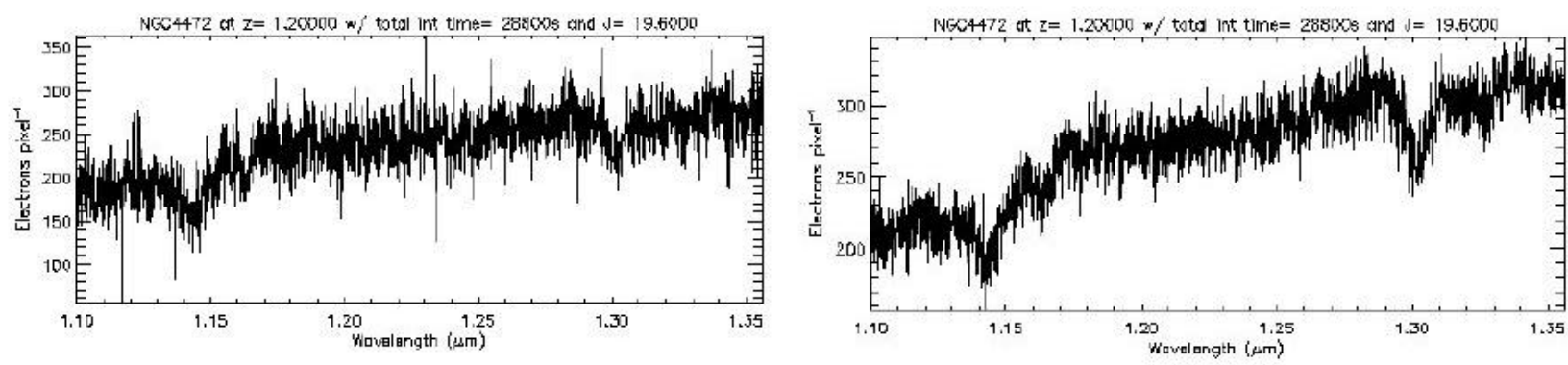

Figure 4. Simulation of a galaxy at $\mathrm{z}=1.2$ with an elliptical $\mathrm{R}^{1 / 4}$ profile, a half-light radius of 0.5 arcseconds, and a Gaussian seeing of 0.5 arc seconds FWHM. The total magnitude of the object is $\mathrm{J}=19.6$ and the integration time is 8 hours. The original spectrum is an integrated spectrum of the nearby elliptical galaxy NGC $4472^{11}$. On the left is shown the simulated spectrum extracted from a slit with a width of 1 arc second and a length of 10 arc seconds. On the right, the simulated spectrum is extracted from an IFU with a hexagonal aperture 2.8 arc seconds in diameter. The signal-to-noise ratio of the slit spectrum is $\sim 10$ while that of the IFU spectrum is 20. In both cases, sky subtraction was "perfect" in that the only impact on the spectrum is increased noise at the position of each night skyline. The difference in the two spectra means that with an IFU, the line strengths (in this case the strong lines of Mgb and $\mathrm{NaD}$ ) can be accurately measured and a velocity dispersion estimated, but this is not the case for the slit.

\subsection{SPECTRAL RESOLUTION AND WAVELENGTH COVERAGE}

Deployable IFUs, through careful design, can be made such that their nominal spectral resolution is two pixels on the detector. With such a sampling, the spectrograph would be as sensitive as possible for the detection of emission lines (especially unresolved or only marginally resolved). To achieve a similar resolution using a slit would require a narrow slit width, thus sacrificing sensitivity for resolution. This also means that the array "filling factor" of the strong nearinfrared night skylines can be minimized using an IFU. 
The spectral resolution should be high enough to resolve the kinematics of galaxies and their building blocks, which according to the hierarchical galaxy formation paradigm should be $\sigma \sim 50 \mathrm{~km} \mathrm{~s}^{-1}$ and below. At the same time, the resolution should also be high enough to permit efficient software suppression of the $\mathrm{OH}$ lines. This suggests that a resolution of 3000-5000 is optimal. For most science questions discussed above having maximum spectral coverage from $0.9 \mu \mathrm{m}$ to $2.5 \mu \mathrm{m}$ is also desirable.

\subsection{PREDICTED SENSITIVITY}

Figure 5 summarizes the $5 \sigma$, 1hour point source sensitivity which should be achievable with KMOS at modest spectral resolution $(\lambda / \Delta \lambda=1000$, with and without adaptive optics), in comparison with $A B=22(K=20)$, elliptical and starburst SEDs at $\mathrm{z}=2.2$. As noted above, KMOS samples various key emission and absorption features, for both old and young stellar populations, which can be detected with good signal to noise ratio using integration times of one to several hours per object. The $5 \sigma, 1$ hour surface brightness sensitivity of KMOS at high spectral resolution (4000) is $A B=18(\mathrm{~K})$ to $\mathrm{AB}=20(\mathrm{~J}, \mathrm{H}) \mathrm{mag} / \mathrm{arcsec}^{2}$. Considering that prominent emission lines $(\mathrm{H \alpha},[\mathrm{OIII}],[\mathrm{OII}])$ have equivalent widths between 10 and $100 \AA$ (line/continuum ratios $\sim 2-10$ ), mapping of the kinematics of emission lines is possible at $>10 \sigma$ per 0.2 " pixel in a 10 hour integration, for objects brighter than $\mathrm{AB} 20(\mathrm{~K})$ to $\mathrm{AB} \sim 22(\mathrm{~J}, \mathrm{H}) \mathrm{mag} / \mathrm{arcsec}^{2}$. Thus, KMOS should be capable of studying both absorption lines in $\mathrm{L}_{*}$ ellipticals at $\mathrm{z} \sim 1$, as well as mapping the kinematics of emission line gas at $\mathrm{z} \sim 1-3$.

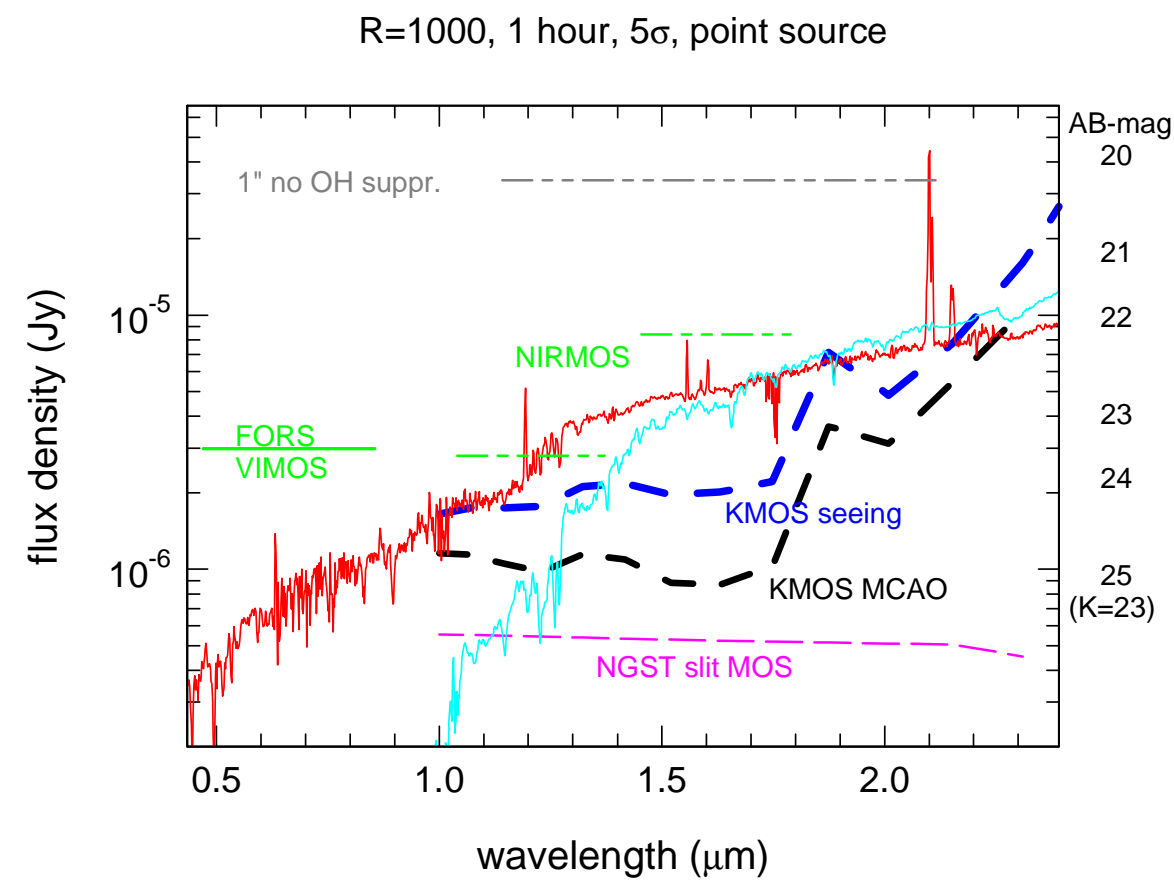

Figure 5. Point source sensitivity ( $5 \sigma$, 1hour integration time at $\lambda / \Delta \lambda=1000,0.5$ " FWHM, blue dashes) of KMOS in comparison to other facilities. The sensitivities were computed for an instrument with $25 \%$ throughput (including detector efficiency), read noise $10 \mathrm{e}$ , dark current $0.05 \mathrm{e} / \mathrm{s}, 0.2$ " diameter pixels, with a standard near-IR atmospheric transmission at T $285 \mathrm{~K}$, and assuming that $\mathrm{OH}$ sky emission is software suppressed by a factor of 50 . For comparison the spectra of a moderately extincted starburst galaxy ${ }^{20}$ (red) and an elliptical galaxy ${ }^{20}$ (light blue) are shown, for a redshift of 2.2 and an AB magnitude of 22. Because of its integral field nature, KMOS gains a factor of $\sim 2$ compared with slit spectrographs, since the effective 'slit' can be optimised for the shape of the source and the seeing (c.f. Figure 3). The lower dashed curve gives the performance of KMOS in conjunction with a high performance (Strehl ratio 0.7 at $2 \mu \mathrm{m}$ ) MCAO system and 0.2 " (software) pixels. 


\section{INSTRUMENT CONCEPT}

KMOS, will be a cooled multi-object, near infrared spectrograph. Within the patrol field of view, small IFU fields can be selected with a cryo-mechanical deployment mechanism allowing flexible and efficient re-configuration of the IFUs. Each of these fields is imaged onto the IFU via dedicated pre-optics. The pre-optics magnifies the field to match the IFU pixel size and can provide a cold stop to suppress stray light. The IFUs dissect the field into individual elements, which are then re-arranged to form part of the spectrograph entrance slit. The spectrograph will be a relatively conventional design, probably based on a reflection grating to achieve the resolution ( $\mathrm{R} \sim 3-5000)$ required for $\mathrm{OH}$ avoidance techniques in the J,H bands. The instrument naturally lends itself to a modular approach where a fixed number of IFUs are fed into a single 'unit spectrograph'. The number of unit spectrographs and IFU feeds can then be duplicated, as funds and mass budgets permit, to obtain the required multiplex capability.

We are currently exploring two different technical solutions for the IFUs based on either image slicers or fibre-lenslets. We are also investigating two different cryo-mechanical deployment strategies adapted to these solutions and will conduct an in-depth trade-off study of the design and functional parameters of KMOS within the constraints of a limited budget, design complexity, and survey efficiency. In the following sections we present the functional requirements and some generic properties of the instrument. The technical IFU studies are discussed briefly in Section 2.3 and in more detail in two accompanying articles.

\subsection{FUNCTIONAL REQUIREMENTS}

Based on the science arguments presented above, we have extracted a set of generic functional requirements (Table 1) for a multi-object near-infrared spectrograph based on deployable integral field units (IFUs), which must be met in order to meet these science goals.

\section{Table 1: KMOS Functional Specification}

\begin{tabular}{|c|c|c|}
\hline PARAMETER & VALUE & REMARKS \\
\hline Wavelength coverage & $0.9-2.5 \mu \mathrm{m}$ & Optional spectral multiplexing of $\mathrm{J} / \mathrm{H} / \mathrm{K}$ bands \\
\hline Number of IFUs & $20-40$ & $\begin{array}{l}\text { trade-off between number of fields, individual IFU size, number } \\
\text { of spectrometer modules and budget }\end{array}$ \\
\hline Patrol field of view & 7.2' diameter & maximum unvignetted field at VLT Nasmyth focus \\
\hline IFU spatial sampling & $0.1 "-0.3 "$ & sampling of MCAO or uncorrected seeing \\
\hline IFU field size & 2" - 4" diameter & trade-off between object size, pixel resolution, and detector size \\
\hline $\begin{array}{l}\text { Positional accuracy of } \\
\text { IFUs }\end{array}$ & $<0.1^{\prime \prime}$ & size of one spatial pixel \\
\hline Stability of IFUs & $<0.01 ”$ & spectral /spatial stability of the system during $3 \mathrm{hr}$ observation \\
\hline $\begin{array}{l}\text { Total re-configuration } \\
\text { time }\end{array}$ & $<10$ minutes & partial re-positioning to be considered \\
\hline Target efficiency & $>75 \%$ & for $\mathrm{N}_{\text {Objects }}=\mathrm{N}_{\mathrm{IFUs}}$ \\
\hline Throughput stability & $<1 \%$ over 1 hour & beam switching can reduce requirement \\
\hline $\begin{array}{l}\text { Minimum target } \\
\text { separation }\end{array}$ & $<20$ " & for observing in clustered fields \\
\hline
\end{tabular}




\begin{tabular}{|l|l|l|}
\hline Spectral sampling & $>2$ detector pixels & Nyquist sampling, could reduce by detector dithering \\
\hline Spectral resolution (R) & $>4000$ & efficient OH line avoidance \\
\hline Scattered light & $<5 \%$ & should not decrease sensitivity by more than $0.5 \mathrm{mag}$ \\
\hline Thermal background & $<5 \%$ of 300K BB & $\begin{array}{l}\text { instrument background < telescope }+ \text { sky background in K and } \\
<\text { OH continuum + detector dark in } \mathrm{J} \& \mathrm{H}\end{array}$ \\
\hline Total system throughput & $>30 \%$ & not including detector \\
\hline
\end{tabular}

\subsubsection{CRYOGENIC OPERATION}

The complete instrument will be cooled to cryogenic temperatures using commercially available closed-cycle coolers. The detectors will be operated at about $77 \mathrm{~K}$; the optimum temperature will be defined when the detector specifications are available. The maximum temperature of the spectrographs can be as high as $140 \mathrm{~K}$ without impairing the instrument performance. The IFU deployment mechanism has to be cooled below $180 \mathrm{~K}$. Depending on the instrument configuration, the complete instrument could be at one temperature, but for technical reasons it may be helpful to keep the deployment mechanism at a higher temperature.

\subsubsection{SPACE AND MASS ENVELOPE}

The volume occupied by the instrument, as well as its mass, depends mainly on the number of unit spectrographs in the instrument and on the collimated beam diameter in the spectrographs. KMOS will be larger and heavier than existing VLT infrared spectrographs, such as ISAAC, but it should be possible to keep the mass and volume within reasonable limits by careful systems engineering using appropriate budget and interface control.

\subsubsection{INSTRUMENT MOUNT}

KMOS will normally be integrating on faint targets for a significant exposure time. Therefore, in order not to compromise the spectral and spatial stability of the IFUs (as specified in Table 1), it is important to avoid internal flexure during rotation of the instrument, which is a particular problem for subsystems mounted in a cryostat. One way to avoid this problem is to mount the instrument on the Nasmyth platform, and either use a special de-rotator (K-mirror), or rotate the IFU deployment mechanism (this is possible only for a fibre coupling between IFUs and spectrographs). An extra tracking sensor would be required to correct for any misalignment between the telescope and instrument. We have developed a preliminary design for a 5-mirror field derotator, which will deliver a 7' field and has a depth (along the optical axis) of only $350 \mathrm{~mm}$. However, our consortium also has a good record of success in controlling flexure for large Cassegrain mounted instruments using careful cryo-mechanical design techniques and active flexure control when necessary.

\subsection{SKY-SUBTRACTION STRATEGIES}

One of the most critical areas of KMOS performance will be the ability to achieve effective sky-subtraction. This is particularly crucial when observing in the near-infrared bands because of the strong and highly variable atmospheric contributions to the background spectrum. Even between the strong $\mathrm{OH}$ lines, the sky continuum flux is comparable to that at optical wavebands ${ }^{12}$. The systematics of sky subtraction (with optical fibres and with IFUs) has been widely discussed. ${ }^{13,14,15}$ Possible sky-subtraction methods for KMOS include:

I. Mean sky method. Dedicating $10 \%$ to $20 \%$ of the IFUs to monitor the sky background in object-free regions of the field.

II. Nodding sky method. Nodding the telescope to position all the IFUs alternately all on objects and all on the sky. 
III. Cross beam-switching method. Positioning $1 / 2$ the IFUs on objects and $1 / 2$ on sky positions, such that each object IFU has an associated sky IFU with the same vector displacement between the sky and object for each pair. Beam switching then occurs such that the sky IFUs become the object IFUs and vice-versa.

IV. Swap beam-switching method. Similar to Method III with half the IFUs on objects and half on the sky, but with no fixed displacement between the sky and object IFUs. After a beam switch, the sky IFUs become object IFUs (observing a different set sources from those at the first-position) and vice-versa.

Each of these methods has a different sensitivity to the parameters of the instrument such as variations in IFU transmittance and PSF variations. Evaluating the optimum method for sky subtraction for the different instrument concepts for KMOS, and quantifying their sensitivity to systematic effects, will be a key outcome of ongoing trade-off studies.

\subsection{TECHNICAL SOLUTIONS}

Existing single-object IFU spectrographs use a variety of techniques to deliver a spatially-resolved spectroscopic capability. ${ }^{15}$ For KMOS we have investigated two technical solutions to the problem of multiple deployable IFUs at cryogenic temperatures. The first is based on fibre-lenslet IFUs which are configured within the focal plane using a cryogenic robot, the second is based on image slicer technology and uses cryogenic pickoff arms to relay the selected targets to a set of fixed IFUs.

\subsubsection{CONCEPT 1}

In this approach the IFUs are composed of 2D arrays of hexagonal lenslets with nearly $100 \%$ filling factor which feed the light into optical fibres. The fibre exits are arranged along the spectrograph entrance slit and the f-ratio of their output beams is defined by a 1D microlens array. Each microlens array, together with its foreoptics, is mounted in a 'spider arm', which can be freely positioned by a cryogenic robot. The input arrays can either be made from monolithic microlens arrays with fibres attached to them, or from tapered fibres where each lens is an integral part of the fibre.

The concept of spider arms, which can be freely positioned, permits the location of these arms on a mildly curved spherical surface. Therefore, the field optics does not have to produce a flat field, although the robot design is much simpler for a flat field. Pre-optics are located in the spider arm between a pickoff mirror and the IFU to provide a cold stop for each IFU and adapt the image scale to the microlens size. A solution to switch between image scales varying by a factor 2 or more (MCAO-oriented and seeing-limited pixel scales) is being explored together with the possibility of having sets of IFUs with different pixel scales and field sizes.

Assuming that the fibres can be made sufficiently long (about $1 \mathrm{~m}$ ) without excessive transmission losses, the focal plane unit and the positioning robot can be conveniently separated from the spectrographs without additional optical elements. This permits the spectrographs to be fixed on the Nasmyth platform and the field rotation to be compensated by turning the focal plane unit. A dedicated IFU with a few arc seconds field size images a bright point source on a CCD to permit continuous measurement and correction of the alignment between instrument and telescope.

The use of fibres to couple the light into the spectrographs potentially allows a larger number of IFUs to be fed into a single spectrograph. This opens up the possibility of providing spectral multiplexing within each spectrograph, using dichroic beamsplitters to feed the light from the J, H \& K bands through a separate grating and camera.

A more detailed description of this concept is given elsewhere. ${ }^{16,17}$

\subsubsection{CONCEPT 2}

An alternative to moving the IFUs about the focal plane is to have a fixed set of IFUs and to relay the light from objects of interest via a set of moveable pickoffs, which patrol the focal plane. In this concept we are investigating a set of individually actuated pickoff probes which feed a set of high-efficiency image slicers. The input beam is guided through the fixed rotation axis of a pickup arm, which also houses the pre-optics and a cold stop. This design keeps the image position and the optical path length constant, irrespective of the IFU field position ad the image slicers re-image the IFU field on the spectrograph entrance slit. 
A field derotator based on a modified K-mirror design is followed by field flattening/telecentric correction optics to produce a flat, telecentric focal plane. Our baseline designs assume either 20 or 32 pickoffs around the edge of the focal plane. The pickoffs are arranged in 3 or 4 layers to provide maximum access to the field without collisions. Each pickoff feeds a $4 \times 4$ arcsec field sampled at 0.2 arcsec/pixel and multiplexed into 5 or 8 spectrographs (Figure 6 ). Because of the desire to obtain uniform spatial sampling and utilise the detector pixels effectively, whilst maintaining 2 pixels per spectral resolution element on the detector, the fore-optics in the IFUs use anamorphic magnification across and along the slices. The set of 5 or 8 identical spectrographs will use either a fixed grating working in multiple orders or a grating wheel. A second wheel will contain the order-sorting filters for each option.

A more detailed description of this concept is given elsewhere. ${ }^{18,19}$

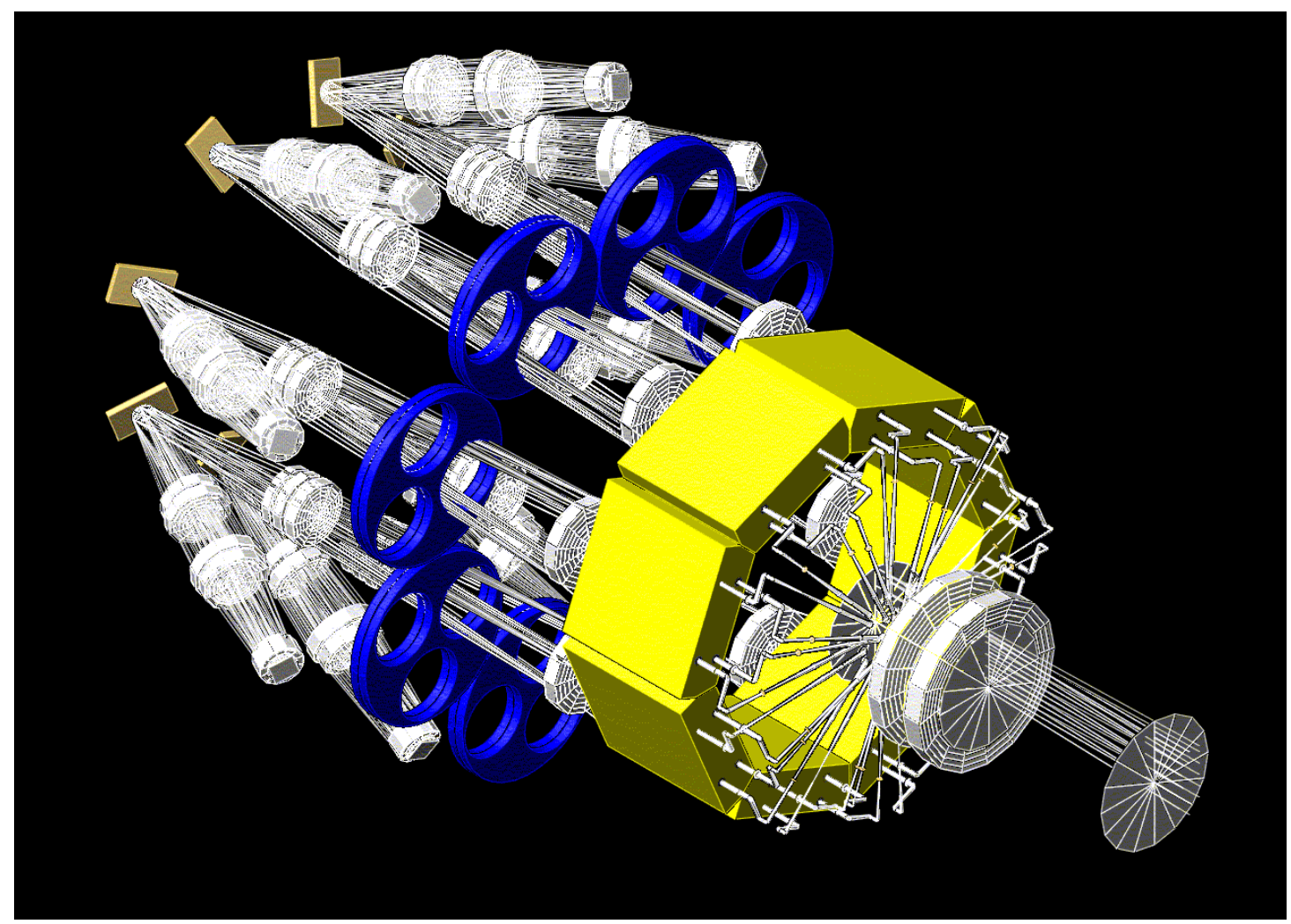

Figure 6. Overall layout of a KMOS instrument based on fixed image slicers fed by cryogenic pickoff arms, including the field flattening optics, 32 pickoffs, 8 IFU modules (each containing 4 IFUs), and 8 spectrographs. The total weight of this concept is estimated to be $3000 \mathrm{~kg}$, within a space envelope of 1.5 metre diameter by 2.5 metre long. The cold mass of the instrument is estimated to be $1000 \mathrm{~kg}$. Liquid nitrogen pre-cool capability will probably be required to achieve respectable cycle times.

\section{SUMMARY}

We have described a proposed $2^{\text {nd }}$ generation instrument (KMOS) for the ESO VLT which will deliver a unique nearinfrared multiple deployable integral field capability for detailed studies of galaxy formation and evolution at high redshift. The science drivers for such an instrument are presented and linked to the functional specification. Detailed technology studies and prototyping of the two proposed solutions for delivering a deployable integral field capability in the infrared are underway, and will be completed by the end of 2003. Assuming that funding is available to begin a preliminary design study at that stage, we estimate that KMOS could be commissioned on the VLT some time in 2009. 


\section{ACKNOWLEDGEMENTS}

We acknowledge input and contributions from all members of the KMOS consortium at the Max-Planck Institut für extraterrestrische Physik, Universitätssternwarte München, Astronomical Technology Centre, University of Durham, University of Oxford and University of Bristol.

\section{REFERENCES}

1. Bershady, M., Lowenthal, J. D., \& Koo, D. C. 1998, ApJ, 505, 50.

2. Rudnick, G., Franx, M., Rix, H.-W., Moorwood, A., Kuijken, K., van Starkenburg, L., van der Werf, P., Roettgering, H., van Dokkum, P., Labbi, I. 2001, AJ, 122, 2205.

3. Drory, N., Bender, R. , Snigula, J., Feulner, G., Hopp, U., Maraston, C., Hill, G. J., de Oliveira, C. Mendes. 2001, ApJL,562, 111.

4. Ferguson, H. C., Dickinson, M., Williams, R. 2000, ARA\&A, 38, 667.

5. Giacconi, R., Rosati, P., Tozzi, P., Nonino, M., Hasinger, G., Norman, C., Bergeron, J., Borgani, S., Gilli, R., Gilmozzi, R., Zheng, W. 2001, ApJ, 551, 624.

6. Daddi, E., Cimatti, A., Pozzetti, L., Hoekstra, H., Roettgering, H. J. A., Renzini, A., Zamorani, G., Mannucci, F. 2000, A\&A, 361, 535.

7. Daddi, E., Broadhurst, T., Zamorani, G., Cimatti, A., Roettgering, H., Renzini, A. 2001, A\&A, 376, 825.

8. Blain, A. W., Kneib, J.-P., Ivison, R. J., Smail, I. 1999, ApJ, 512, 87.

9. Le Fevre, O., Abraham, R., Lilly, S. J., Ellis, R. S., Brinchmann, J., Schade, D., Tresse, L., Colless, M., Crampton, D., Glazebrook, K., Hammer, F., Broadhurst, T. 2000, MNRAS, 311, 565.

10. Ivison, R. J., Smail, I., Frayer, D. T., Kneib, J.-P., Blain, A. W. 2001, ApJ, 561, 45.

11. Kennicutt, R. C., Jr. 1992, ApJS, 79, 255.

12. Maihara, T., Iwamuro, F., Yamashita, T., Hall, D.N.B., Cowie, L.L., Tokunaga, A.T. \& Pickles, A. 1993, PASP, $105,940$.

13. Wyse, R.F.G. and Gilmore, G. 1992, MNRAS 257, 1.

14. Cuby, J-G and Mignoli, M. 1994, SPIE 2198, 98.

15. Allington-Smith J.R. and Content, R. 1998, PASP 110, 1216.

16. Genzel, R., Hofmann, R., Tomono, D., Thatte, N., Eisenhauer, F., Lehnert, M., Tecza, M., and Bender, R. 2001, A cryogenic near-infrared, multi-object spectrometer for the VLT, talk given at the ESO workshop on scientific drivers for ESO future VLT/VLTI instrumentation, astro-ph/0108318.

17. Tomono, D., Weisz, H. \& Hofmann, R. 2002, these proceedings.

18. Wright, G., Ivison, R., Hastings, P., Wells, M., Sharples, R.M., Allington-Smith, J.R. and Content, R. 2001, Multiple integral field spectroscopy, talk given at the ESO workshop on Scientific drivers for ESO future VLT/VLTI instrumentation, astro-ph/0202306.

19. Ramsey-Howat, S.K., Sharples, R.M., Wright, G.S., Hastings, P.R., Wells, M., Cunningham, C.R., Schmoll, J., Content, R. \& Robertson, D. 2002, these proceedings.

20. Kinney, A.L., Calzetti, D., Bohlin, R.C., McQuade, K., Storchi-Bergmann, T. \& Schmitt, H.R. 1996, ApJ, 467, 38. 